\title{
Specificity and flexibility of social influence on spatial choice
}

\author{
Michael F. Brown ${ }^{1} \cdot$ Marie E. Saxon ${ }^{1} \cdot$ Kelsey A. Heslin ${ }^{1}$ \\ Published online: 21 March 2018 \\ (C) Psychonomic Society, Inc. 2018
}

\begin{abstract}
Rats searched for food in a situation that allowed them to determine which locations contained food after searching a small number of them, but not which of the baited locations contained more-preferred food rather than a less-preferred food. During some experimental trials, the latter information was available from the choices of model rats making choices together with the subject rats, because some of the model rats tended to choose the locations baited with more-preferred food. On the surface, the results suggest that social influence specified the locations of more-preferred food to the subject rats. However, more detailed analysis and data from a second experiment indicate that the social influence can be explained by a general tendency to approach another rat making choices, acquired if rats are exposed to a contingency between social approach and increased foraging success.
\end{abstract}

Keywords Social influence $\cdot$ Social cognition $\cdot$ Spatial cognition

Animals foraging in groups influence each other in ways that can increase or decrease the foraging efficiency of any particular individual (see Giraldeau \& Caraco, 2000; Ward \& Webster, 2016, for reviews). In many cases, individuals with more information influence the behavior of those with less information (e.g., Vickery, Giraldeau, Templeton, Kramer, \& Chapman, 1991). Laboratory models of such social influences include studies of pairs of rats searching for food among spatial locations (Brown, 2011), which is the context of the present study.

The mechanisms of social influences can be characterized on a continuum with respect to how flexibly social cues influence behavior. One end of the continuum is an inflexible tendency to mimic the behavior of conspecifics and/or to approach them. For example, Galef and Whiskin (2008) showed that rats' tendency to choose foods matching the flavor of food eaten by a demonstrator rat occurs even for foods that have become otherwise aversive. Thus, at least under some conditions, social influences on food preferences supersede personal experience that otherwise makes the food unpalatable. Similarly, when two rats choose spatial locations in a radial-

Michael F. Brown

michael.brown@villanova.edu

1 Department of Psychology, Villanova University, 800 E. Lancaster Ave., Villanova, PA 19085, USA arm maze, the presence of one rat in or near a maze arm facilitates choice of that location by a second rat (Brown, 2011). This occurs despite the absence of food in that location (because the first rat has depleted it). In fact, the second rat avoids locations previously chosen by the first rat if the first rat is no longer present there (Brown, Farley, \& Lorek, 2007), indicating that the presence of the first rat elicits approach in a relatively inflexible manner.

Under other conditions, however, social influences appear to be controlled by specific social stimuli that provide information relevant to the foraging requirements and which are used flexibly to enhance foraging efficiency. Such influences are often referred to as "public information" in the behavioral ecology literature (e.g., Danchin, Giraldeau, Valone, \& Wagner, 2004). In laboratory foraging tasks like the radialarm maze or the pit maze used in the present study, social influences could be based on cues corresponding to another rat discovering the location of food or procuring and eating the food. Evidence that such cues (rather than the mere presence of another rat) are critical include the finding that rats are more likely to later visit a location in the radial-arm maze previously visited by another rat if the other rat found a large cache of food there rather than a small, depletable cache (Brown, Prince, \& Doyle, 2009) or if it found a preferred food rather than a less preferred food type (Brown et al., 2008). These findings indicate that specific physical or behavioral cues provided by one rat convey information to another rat about the presence or quality of food in a location. 
In between inflexible social influences on spatial choice such as those produced by social affiliation tendencies and flexible use of more specific social cues, there is a continuum of moreor-less flexible and more-or-less specific forms of social influence. In naturally occurring behaviors, a wide scope of conditions appear to modulate the relative control by "personal" versus "public" information, such that the same animal uses these two information sources to very different degrees under different conditions (e.g., Giraldeau, Valone, \& Templeton, 2002; Rieucau \& Giraldeau, 2011). The extent to which rats are influenced by the spatial choices of foraging partners in laboratory tasks can be modulated by the validity of the information provided by the other rat (e.g., Laland \& Plotkin, 1990) or by the value of the information to the receiving rat (Galef, Dudley, \& Whiskin, 2008). Here, social influence is not very specific or flexible locally but is globally modulated depending on the conditions experienced by the animal over relatively large periods of time (i.e., during its experimental experience).

This paper describes an unexpected result that provides an opportunity to examine the flexibility and specificity of social influence. In the "pit maze" (Keller \& Brown, 2011), rats search for caches of food hidden in some of the 25 covered pits configured as a $5 \times 5$ matrix in an open field arena. Previous experiments show that social influences between two rats simultaneously searching for food in this task vary in accordance with the degree to which an informed "model" rat provides information about the location of the food to a less informed subject rat (Bisbing, Saxon, Sayde, \& Brown, 2015).

Brown et al. (2015) used a procedure in which partial information about the baited locations was available to the subject rats from the outcome of their own choices, but more complete information was available from the choice behavior of model rats that had additional information about the location of the hidden food. This was arranged using sets of five baited locations that were all on one of the two sides of the arena. Given that all five baited locations were among the 10 locations on one side of the arena, choice of a baited pit reveals that the remaining baited pits are on that same side. Choice of an unbaited pit does not necessarily mean that the baited pits are on the other side of the maze, but it does indicate they are more likely to be on the other side of the maze than on the side of the chosen (and unbaited) location. Because each model rat found pellets in the same pits over trials, they had information about not only the side of the arena that was baited but also the specific pit locations that were baited. When a model rat and subject rat were tested together under these conditions, there was evidence that both the "personal information" provided by the outcome of the subject rats' own choices and the social information provided by the choices of the model rats controlled the spatial choices of the subject rats.

The first experiment reported below was intended as a replication and extension of the Brown et al. (2015) work described above. The extension involves the difference between the information provided by the outcome of rats' own choices and that provided socially. In Brown et al.'s experiment, that difference was a matter of resolution. The "personal information" obtained from the outcome of a subject rat's own choices determined the side (or general area) of the maze that was baited, whereas the social information cued the specific pits that were baited. In the present experiments, the personal information similarly provides information about the side of the maze where baited pits are located. However, in this experiment, all 10 locations on the baited side are baited. Five of them are baited with grain pellets and the other five are baited with sucrose pellets (known to be preferred over grain pellets; e.g., Brown et al., 2008). The spatial configuration of the five grain-baited locations and five sucrose-baited locations on the baited side is unpredictable from the subject rats' perspective, but is consistent over trials for any particular model rat. Thus, in the present experimental design, personal information provides the location of baited pits (where food is hidden), whereas information available exclusively from social cues can indicate the type or quality of hidden food (what is there).

The unexpected result was an individual difference among the model rats in whether they exhibited a tendency to choose the sucrose-baited locations. About half of them did, but half of them did not. Although this reduced our ability to address the original question, it also provided an opportunity to examine the specificity and flexibility of the model rats' influence on the choices of the subject rats. The social influence found in laboratory spatial search tasks like that described above could be based on a relatively inflexible tendency to "follow" other rats. Alternatively, it could be based on flexible use of cues provided by the model rats that correspond to finding food in a location or - in this case - finding preferred food in a location. To the extent that the social cues are specific and control the choices of the subject rats in a flexible manner, subject rats should be socially influenced to a greater extent by model rats that reveal the location of the preferred food than by model rats that do not.

\section{Experiment 1}

\section{Method}

Subjects Twenty-four experimentally naïve Sprague-Dawley male rats were obtained from Harlan Sprague-Dawley (Indianapolis, IN). They were housed in pairs in $45 \mathrm{~cm} \times$ $24 \mathrm{~cm} \times 20 \mathrm{~cm}$ (tall) cages on a 12:12 hour reversed light:dark (LD) cycle. The rats were tested during the dark phase of the cycle. Rats were food deprived and were maintained at $90 \%$ of their free feeding weights (determined by individual free-feeding weights prior to the experiment, combined with growth curves provided by the vendor). They had ab libitum access to water. They were randomly assigned cage mates (pairs of two) when 
they arrived from the vendor. The experiment began when the rats were 3 months old. Each rat was assigned to be either a model rat or a subject rat; one rat in each pair of cage mates was randomly assigned as a model and the other was assigned as a subject.

Apparatus Testing took place in a $1.17 \mathrm{~m} \times 1.17 \mathrm{~m}$ arena enclosed by $14-\mathrm{cm}$ tall walls and painted flat black (see Figs. 1 and 2 of Keller \& Brown, 2011). A rat cage was attached to an outside wall of the arena and served as the start box. A guillotine door, controlled by a string-and-pulley system, determined access to the arena. A matrix of equally spaced $(20.0 \mathrm{~cm}$ center-tocenter) "pits" defined the locations where rats searched for hidden food pellets. The pits were constructed of plastic funnels placed inside of plastic drinking cups. The tops of the drinking cups were level with the floor of the arena. The pits were $5.0 \mathrm{~cm}$ deep, $7.6 \mathrm{~cm}$ in diameter at the top, and $1.3 \mathrm{~cm}$ in diameter at the bottom. The bottom of each pit was a mesh screen where food pellets could be placed. This screen was at base of the funnel stem. Additionally, there were "sham" pellets placed underneath the mesh floor of each pit in the bottom of the cup. These were present to control any odor cues that the pellets may provide. Each pit contained eight to 10 sham sucrose pellets throughout the experiment.

A second funnel was placed inside each pit to function as a lid. Rats learned to lift the lid to gain access to the pellets that might be located inside the pit. These covers were attached to a string system that ended with $14 \mathrm{~g}$ weights (hidden underneath the apparatus), thereby resulting in the cover being retracted into the pit when a rat was not lifting it.

Training phases Rats were first exposed to the apparatus and to the general features of the procedure before the start of the experiment. Each pair of cage mates experienced three daily, 10 minute exposures to the arena during which pellets (grain and sucrose) were scattered on the floor. For model rats, these exposure sessions were followed by the training trials described in the next paragraph. For subject rats, four additional days of exposure training to the maze occurred weeks later, as the model rats were completing the training trials.

In the case of model rats, the exposure sessions were followed by training trials intended to allow the model rats to learn the locations of baited pits. The set of 10 pits assigned to each model rat contained food pellets (five baited with grain, five baited with sucrose; see below for details of assignments). The first 3 days of model rat training consisted of 5-minute trials in which visual cues to food location were available. These visual cues included pellets scattered around the outside of each baited pit and the lids on the pits being ajar. After all models rats were investigating (i.e., sticking their heads into) all 10 pits on their assigned side, the trial duration was decreased to 2 minutes for the remainder of model rat training. In addition, the visual cues corresponding to the location of baited pits were no longer present.
Experimental phase Trials of the experiment were of two kinds: together trials and alone trials. In together trials, subject rats were paired with model rats (pairing varied over trials as detailed below) and the two rats entered the maze together. In together trials, the baited pit locations were determined by the assignments of the model rat in the pair (see below). In alone trials, each subject rat and model rat entered the maze individually. During these trials, model rats found food in their consistent assigned set while subject rats were randomly assigned a particular model rat's assigned set.

Prior to each trial, pits were baited with sucrose and grain pellets in accordance with a given model rat's assigned set of baited pits. Each model rat's $(n=12)$ assigned set consisted of 10 pit locations where it found food consistently throughout the experiment. For each model rat, the 10 baited locations were either all on the east side of the maze ( $n=6$ rats) or all on the west side of the maze $(n=6)$; the five pits at the boundary between the two arena sides were never baited. Additionally, five of the 10 locations were assigned to be baited with sucrose pellets, while the other five locations were baited with grain pellets. The type of food in each baited pit location was determined randomly for each model rat, with the constraint that it was counterbalanced across model rats. That is, each pit location was baited with sucrose for $25 \%$ of the models, grain for $25 \%$ of the models, and was on the unbaited side of the arena for the remaining 50\% of the models (ignoring the five pits forming the boundary of the two arena sides, which were never baited for any rat). Each baited pit contained five to eight pellets.

At the beginning of each trial, the rat or pairs of rats were placed in the start box (which was attached to the apparatus at the midline of the boundary between the arena sides). The door blocking access to the maze was opened, and once the rat(s) entered the maze a timer of 1-minute would begin. This timer was started when both rats' heads were in the maze. The rats were removed from the arena after this 1-minute time period was over. Trials were recorded with a video camera that was mounted directly above the maze.

Subsequently, rats were tested during two trials per day for 5 days a week. These trials were structured in four blocks of 12 trials each. In each block, rats were tested alone (alone trials) for six trials and with another rat for six trials (together trials). The order of these matchups was randomized within each of two 2-block chunks, wherein each subject rat was paired with each model rat twice (once for together trials and once for alone trials).

\section{Results}

The first set of results of interest is the tendency of model rats and subject rats to choose pits on the baited rather than unbaited side of the arena. The measure of this tendency was the proportion of choices made to pits on the baited area side 


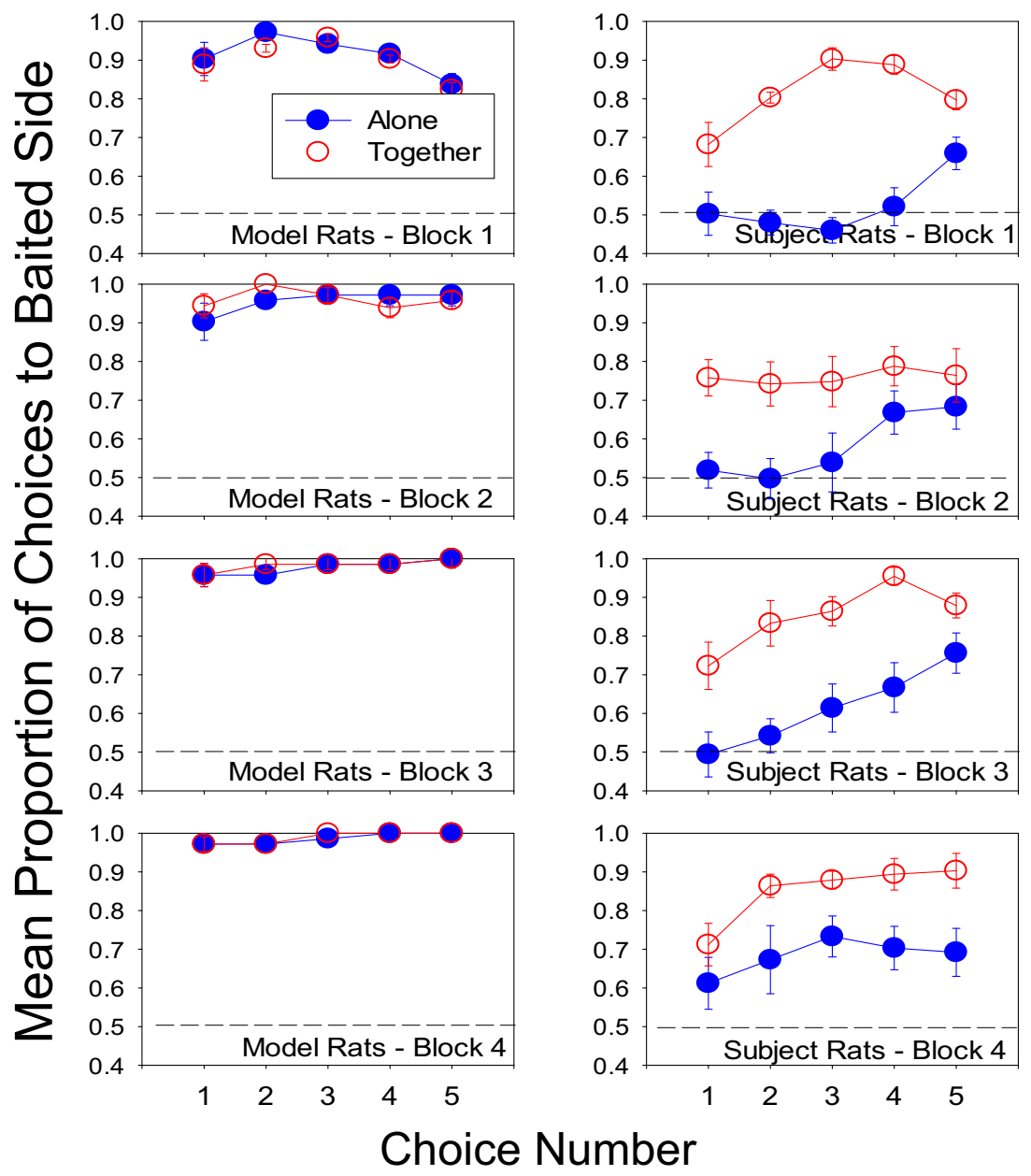

Fig. 1 Tendency to choose pits on baited side of arena in Experiment 1. Error bars show one standard error of the mean

(choices to pits in the middle column of pits between the two sides rarely occurred and were not included). The mean proportions are shown in Fig. 1 for model rats (left panels of figure) and subject rats (right panels). In the case of the model rats, the proportions were very often 1.0 or very close to 1.0 . Thus, in many cases, these data violated assumptions required for proper application of analysis of variance (ANOVA), so we did not attempt to analyze the effects of all the factors (trial block, choice number, and trial type) represented in Fig. 1 (left panels). It suffices to point out that the model rats apparently exhibited a strong tendency to choose pits on the baited rather than unbaited side of the arena.

Because one of the subject rats often made only two or three choices per trial (and therefore had some trial blocks with no fourth or fifth choices), its data are not included in this analysis (its data are included in the subsequent analyses that collapse over choice numbers).

A 2 (trial type: together vs. alone trials) $\times 4$ (trial block) $\times 5$ (choice number) ANOVA was used to compare the tendency of subject rats to choose pits on the baited side of the arena in together and alone trials as a function of trial type over the course of the four blocks of trials and over the first five choices made within trials. This ANOVA revealed significant effects of block, $F(3,30)=8.50, p=.001, \eta_{\mathrm{p}}{ }^{2}=.480$; choice, $F(4,40)$ $=9.25, p<.001, \eta_{\mathrm{p}}{ }^{2}=.481$; trial type, $F(1,10)=141.95, p<$ $.001, \eta_{\mathrm{p}}{ }^{2}=.934$, and a significant interaction of Trial Type $\times$ Choice, $F(4,40)=3.46, p<.05, \eta_{\mathrm{p}}{ }^{2}=.257$ (ANOVA factors are reported in this paper only if they produced significant, $p<$ .05 , effects). Perhaps most critically, subject rats had a stronger tendency to choose locations on the correct side of the arena during together trials than during alone trials.

The second set of results of interest is the tendencies of model and subject rats to choose pits baited with the preferred sucrose pellets over pits baited with grain pellets. Two 2 (trial type: together vs. alone trials) $\times 4$ (trial block) $\times 5$ (choice number) ANOVAs were used to compare this tendency in together and alone trials over the course of the four blocks of trials and over the first five choices made within trials. The measure of this tendency was the proportion of choices made on the baited side of the arena that were to pits baited with sucrose (rather than those baited with grain). The mean proportions are shown in Fig. 2 for model rats (left panels of figure) and subject rats (right panels). In the case of model rats (Fig. 2, left panels), the ANOVA revealed no significant 

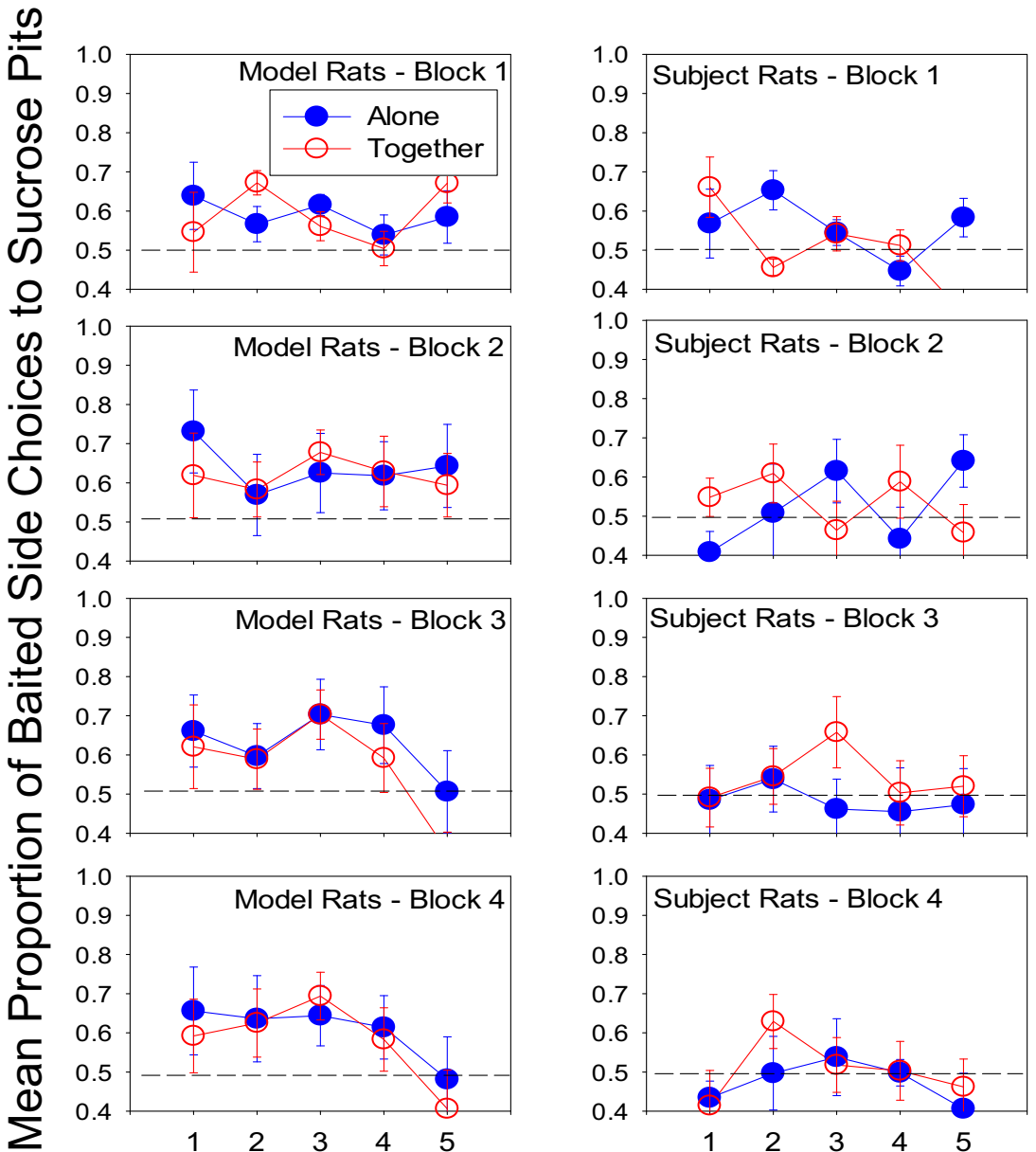

\section{Choice Number}

Fig. 2 Tendency to choose sucrose pits over grain pits in Experiment 1. Error bars show one standard error of the mean

effects, although the trial type factor approached significance, $F(1,11)=4.468, p=.056, \eta_{\mathrm{p}}{ }^{2}=.288$. In the case of subject rats (Fig. 2, right panels), the ANOVA revealed no significant effects.

It is not surprising that subject rats did not show a tendency to choose pits baited with sucrose over pits baited with grain, given that social information is necessary for discriminating the two kinds of locations and that the model rats, as a group, did not demonstrate a strong tendency to selectively visit the pits baited with sucrose. It remains possible, however, that some model rats did selectively visit pits baited with sucrose and that social influences might be revealed if subject rat choices are examined separately for trials involving model rats that did exhibit such a tendency. Table 1 shows the mean proportion of each model rat's choices on the baited side of the apparatus that were to sucrose pits. Means were calculated from the proportions for each choice number during each trial block, separately for the alone and together trials for each model rat. A set of $t$ tests was used to define which model rats had a tendency to choose sucrose pits over grain pits in each of the two trial types (see Table 1). The mean proportions of
Table 1. Individual model rat tendencies to choose sucrose pits mean proportion of baited-side choices to sucrose pits ( $t$ value for mean proportion vs. 0.5$)$ ordered by mean proportion for together trials

\begin{tabular}{lll}
\hline & Together trials & Alone trials \\
\hline $12 \mathrm{~N}^{* *}$ & $.81(7.35)^{*}$ & $.83(6.13)^{*}$ \\
$2 \mathrm{~N}^{* *}$ & $.72(4.70)^{*}$ & $.74(4.84)^{*}$ \\
$1 \mathrm{~N}^{* *}$ & $.69(3.94)^{*}$ & $.70(3.27)^{*}$ \\
$8 \mathrm{~N}^{* *}$ & $.68(2.20)^{*}$ & $.66(2.68)^{*}$ \\
$6 \mathrm{~N}^{* *}$ & $.64(2.21)^{*}$ & $.70(3.92)^{*}$ \\
$7 \mathrm{~N}$ & $.57(1.26)$ & $.60(1.12)$ \\
$3 \mathrm{~N}$ & $.56(1.02)$ & $.55(<1)$ \\
$11 \mathrm{~N}$ & $.54(<1)$ & $.52(<1)$ \\
$5 \mathrm{~N}$ & $.52(1.94)$ & $.72(4.74)^{*}$ \\
$4 \mathrm{~N}$ & $.49(<1)$ & $.54(<1)$ \\
$9 \mathrm{~N}$ & $.45(<1)$ & $.51(<1)$ \\
$10 \mathrm{~N}$ & $.33(-2.80)^{*}$ & $.30(<1)$ \\
\hline
\end{tabular}

Note. Mean proportions were calculated over four trial blocks and five choices ( 19 degrees of freedom). $* p<.05$. **rat is defined by these results as "good" model 
choices to sucrose pits for each model rat in the alone and together trials were compared to chance (.50). Five of the 12 model rats were making choices to sucrose locations significantly more than grain locations during both alone trials and together trials. "Good" model rats are hereby defined as the five rats that demonstrated a significant tendency to choose pits baited with sucrose in both alone trials and together trials. This allows examination of choices by the subject rats as a function of whether they are in the presence of model rats that exhibited a tendency to choose pits baited with sucrose ("good model rats") or did not ("bad model rats").

Figure 3 (top panel) shows the tendencies of the five "good" model rats and the seven "bad" model rats to choose sucrose pits over grain pits. A 2 (model type: "good" vs. "bad" models as a between-subjects factor) $\times 4$ (trial block) ANOVA revealed a significant effect of model type, $F(1,10)=13.8, p<.01, \eta_{\mathrm{p}}{ }^{2}=$ .58. There was also an interaction between effects of model type and trial block, $F(3,30)=3.7, p<.05, \eta_{\mathrm{p}}{ }^{2}=.27$, apparently due to the increase in choice of sucrose pits by good model rats, but not by bad model rats, over trial blocks.

Figure 3 (middle panel) allows comparison of the tendencies of subject rats to choose sucrose pits over grain pits during trials in which they were tested together with "good" model rats and those in which they were tested with "bad" model rats (the tendencies during alone trials are also shown). A 2 (partner type: "good" vs. "bad" models) $\times 4$ (trial block) ANOVA revealed a significant effect of partner type, $F(1,11)=35.47, p$ $<.001, \eta_{\mathrm{p}}{ }^{2}=.763$.

The bottom panel of Fig. 3 shows the mean proportions of choices that were made during together trials to pits that had been visited earlier during the trial by the other rat. The tendency to choose pits visited by the other rat is shown separately for good and bad model rats (green data points) and also for subject rats when tested with either good or bad model rats (red data points). A series of three ANOVAs evaluated the relevant comparisons among these tendencies. First, a rat type (model vs. subject rats) $\times$ trial clock ANOVA confirmed that subject rats choose pits earlier visited by model rats to a greater degree than model rats choose pits earlier visited by subject rats, $F(1,22)=30.25, p<.001, \eta_{\mathrm{p}}^{2}=.579$. A Model Type (good vs. bad model rats) $\times$ Trial Block ANOVA did not reveal any significant differences in the tendency of good versus bad model rats to visit pits that were earlier visited by the subject rats. More critically, A Model Type (good vs. bad model rats) $\times$ Trial Block ANOVA did not reveal any significant differences in the tendency of subject rats to visit pits as a function of whether they were being tested together with good or bad model rats.

\section{Discussion}

In agreement with the results of Brown et al. (2015), both personal information and social information controlled the
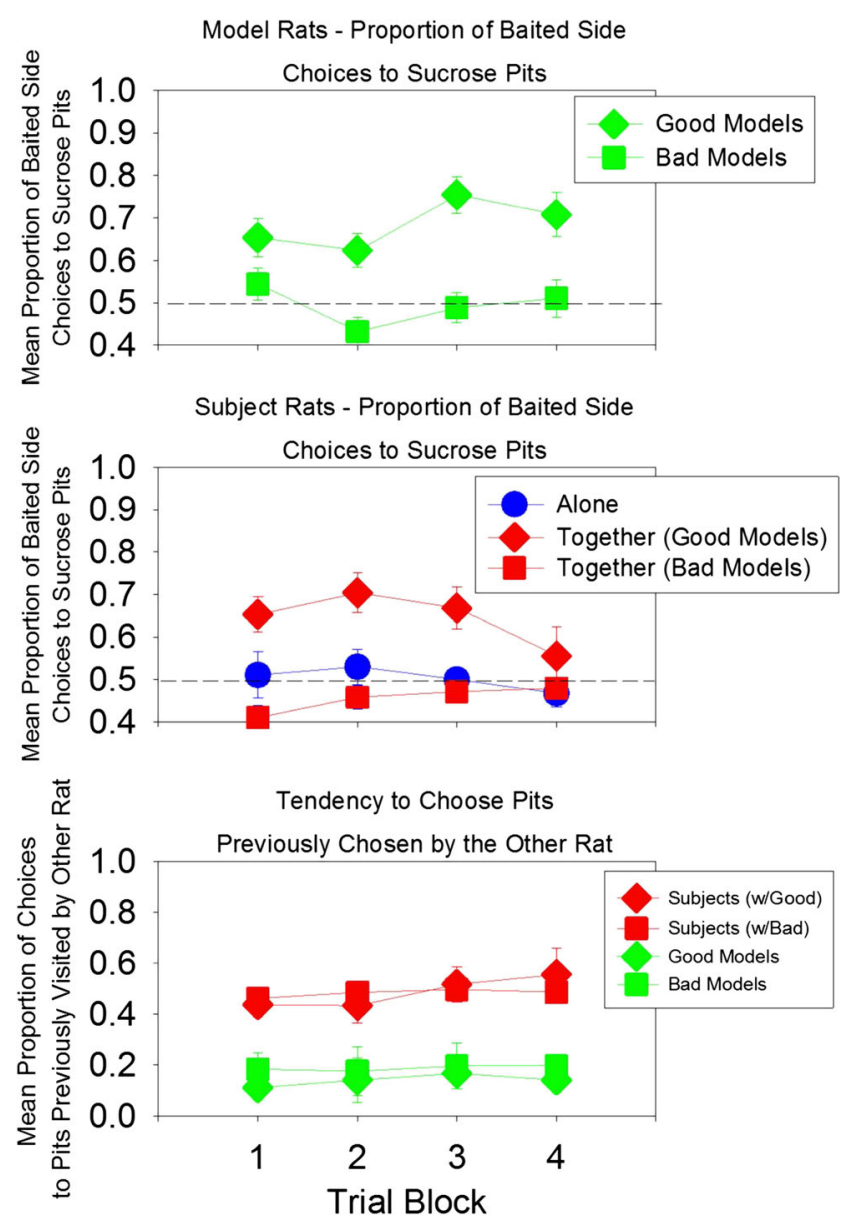

Fig. 3 Comparison of good versus bad model rats in terms of model rat tendency to choose sucrose pits (top panel), subject rats tendency to choose sucrose pits in alone trials and in together trials with good versus bad model rats (middle panel) and rats' tendency to choose pits that were previously chosen by the other rat (bottom panel). Error bars show one standard error of the mean

side of the arena in which choices were made by subject rats. In alone trials, there was a tendency to choose pits on the baited arena side that increased both over choices within each trial and as a function of experience over trials. This indicates that the contingency between the bait status of pits chosen and the location of remaining baited pits is effective; it comes to control choices as the rats acquire experience with that contingency over the course of the experiment and as they make choices within each trial.

At the same time, social information also controlled choices. This is shown by the enhanced tendency to choose pits on the baited side of the arena in the together trials, relative to the alone trials. It is noteworthy that even on the first choice made in together trials, when no information is available from the outcome of previous choices by the subject rat itself, choices were to pits on the baited side of the arena substantially more than expected by chance.

However, in contrast to the pattern of results reported by Brown et al. (2015), social information provided by the model 
rats (considered all together) did not allow the subject rats to discriminate among the pits on the baited side of the arena. Brown et al. found that, when tested together with model rats, subject rats selectively chose baited pits on the baited side of the arena over unbaited pits on the baited side of the arena. The data shown in Fig. 2, on the other hand, provide no evidence that rats use social information to selectively choose pits baited with sucrose over pits baited with grain pellets. The direct comparison of subject rat performance in the two experiments is not appropriate, however, because only some of the model rats in the present experiment demonstrated a tendency to choose pits baited with sucrose over pits baited with grain pellets.

When the analysis is restricted to together trials involving model rats that did exhibit a tendency to choose sucrose baited pits over grain baited pits ("good" model rats), the subject rats in this experiment, like those in the earlier experiment, exhibited a tendency to discriminate among the pits on the baited side of the arena. This result suggests that choices made by the good model rats provide informational cues, in the sense that they control choices in the subject rats because they indicate whether the more highly valued sucrose pellets or less valued grain pellets are available in a particular location. An alternative to the idea that choices made by good model rats provide informational cues is that rats elicit social affiliative tendencies in other rats. That is, subject rats may be drawn toward model rats regardless of any information provided by their choices. Approach, in turn, could make choice of pits just chosen by the model rat more likely, simply because of their proximity when the subject rat makes its next choice. If so, approach would thereby result in selective choice of sucrose-baited pits only if the observed rat selectively chooses them.

The social affiliation interpretation of social influence on choice of sucrose versus grain locations by the subject rats is consistent with the fact that subject rats were just as likely to visit the pits on the baited side of arena that had earlier been visited by the bad model rats as they were to visit pits that had earlier been visited by the good model rats (see Fig. 3, bottom panel). On the other hand, the fact that subject rats were much more likely to visit pits that had earlier been visited by model rats than model rats were to visit pits that had earlier been visited by subject rats (see Fig. 3, bottom panel) supports an informational interpretation of social influence. The asymmetry of social influence between model rats and subject rats indicates that subject rats chose locations that were chosen by model rats because model rats choose pits on the baited side of the arena and perhaps also because some model rats more often chose pits baited with the preferred food type. However, there is no evidence that subject rats discriminate between those model rats more likely to choose sucrose locations over grain locations versus those that choose baited locations indiscriminately. Nor is there any evidence that they can discriminate what kinds of food a model rat finds in, or eats from, a particular location.

\section{Experiment 2}

Experiment 2 examined the nature of the cues that control social influence on spatial choices in the experimental context used in Experiment 1. Experiment 1 indicates that social influence on choices occurs when rats experience other rats making informative choices (i.e., subject rats), but does not occur when rats experience other rats making choices that provide little or no information about the location of food (i.e., model rats). However, the nature of the cues corresponding to model rats that provided information to subject rats is unclear.

To examine the nature of the social cues used by subject rats, the protocol of Experiment 1 was modified in two experimental conditions, such that a subject rat was restrained and could only observe as the model rat made choices. In one condition (delayed release), the subject rat was released to begin making choices just as the model rat made its second choice. In a second condition (delayed release and removal), the subject rat was also released to begin making choices after the model rat made its second choice, but the model rat was first removed from the arena. A third condition was equivalent to the together condition of Experiment 1 (no restraint of the subject rat). Because this experiment examines the cues responsible for the phenomena observed in Experiment 1, we used the same rats assigned as models and subjects as they were in the earlier experiment.

The tendency to choose pits that were chosen by the model rat (as its first or second choice) were compared in these three conditions and for trials involving the good and bad model rats. Two kinds of cues that could be responsible for social influences on choices by the subject rats were considered. The first was cues that correspond to the physical presence of the model rat. Alternatively, choice of a location by the model rat could alter a representation that corresponds to the location (e.g., a memory of the rat being there; a value associated with the spatial location itself). Choices made by the subject rat when the model rat remained physically present in a chosen location and choices made when the model rat had been removed were compared. The comparison was intended to reveal which of these kinds of cues are effective. Furthermore, comparisons involving the good and bad model rats provided an additional test of whether subject rats discriminate between model rats according to whether they preferentially choose sucrose locations or can discriminate the type of food found by the observed model rat during a particular choice. 


\section{Method}

Subjects The same 24 rats that participated in Experiment 1 were tested. Approximately 4 months elapsed between the experiments, during which the rats were maintained on a free-feeding schedule. One week before the start of Experiment 2, they were put on a food-restricted diet and thereafter maintained at the same level of food deprivation as in Experiment 1 (90\% of free-feeding weight, as determined during the free-feeding between the two experiments, corrected for growth curves).

Apparatus The apparatus was the same as used in Experiment 1 , with one modification. The pit that had been in the center of the arena for Experiment 1 was replaced with a circular cage. The bottom of the cage was a round, clear plastic disc $21 \mathrm{~cm}$ in diameter and $2 \mathrm{~cm}$ above the floor of the arena. A wire mesh basket (of approximately the same diameter and $14 \mathrm{~cm}$ tall) formed the remainder of the cage when it was placed upsidedown on the cage bottom. It will be referred to below as the "observer cage" because its purpose was to hold subject rats such that they could observe a model rat in the arena while preventing the subject rat from making choices, as shown in Fig. 4.

Procedure The baited pit assignments for model rats and other details of the procedure not mentioned below were the same as in the experimental phase of Experiment 1. There were three kinds of experimental trials. Together trials were identical to the together trials of Experimental 1, except that the observer cage was present in the center of the arena throughout the trials (thus, there were 24 rather than 25 pits in the arena). Delayed release (DR) trials differed from together trials in that the subject rat was placed in the observer's cage and the cage cover put in place prior to each trial. The model rat was then placed in the start box and released to begin the trial. The model rat was allowed to make two choices and then, as soon as the model rat made its second choice, the subject rat was released from the observer cage by removing the cage cover (using the handle on top of the cage cover). Delayed release and model removal (DRMR) trials were identical to DR trials except that the model rat was removed from the maze as soon as it made its second choice, and the subject rat was then released from the observer cage.

The rats were tested in three blocks of 11 trials each, with one or two trials conducted per day (generally 5 days per week). Testing always occurred during the dark phase of the rats' LD cycle. If two trials were conducted on a particular day, they were separated by at least 2 hours for any particular rat. In each trial block, each subject rat was tested during one trial with each of the model rats (except its cage mate; cage mates were not tested together in this experiment). In each trial block there were either three or four trials of each type (together, DR, and
DRMR) and, over all three trial blocks there were 11 of each type for each rat. Within these constraints, the trial type was assigned randomly for each block of trials.

\section{Results}

Figure 5 shows the proportions of choices made to pits on the unbaited side of the arena, pits baited with grain pellets, and pits baited with sucrose pellets during the three types of trials among the first five choices made by the subject rat (as in Experiment 1, choices to the four pits between the arena sides were very rare and not included). Data are separated for trials involving the "good" (left panels of figure) and "bad" (right panels) model rats (classified from Experiment 1). The top panels show choices made by the model rats. The bottom panels show choices made by the subject rats when they are tested with the good and bad model rats (bottom-left and bottom-right panels, respectively). It should be noted that there are twice as many pits on the unbaited side of the arena (10) as there are pits baited with grain (5) or with sucrose (5) on the baited side of the arena. This is represented by the dotted reference lines in the figure depicting the proportions expected if the rats choose pits randomly.

Model rats in both categories chose pits on the unbaited side of the arena less than expected by chance in all three trial types: for good models, $t \mathrm{~s}(4) \geq 44.8$; for bad models, $t \mathrm{~s}(6) \geq$ 21.3. Good model rats were more likely to choose sucrosebaited pits than grain-baited pits in all three trial types, $t \mathrm{~s}(4)>$ 2.9, but bad model rats did not exhibit a significant tendency to choose sucrose-baited pits over grain-baited pits in any of the experimental conditions, $t \mathrm{~s}(6)<1.4$ (the proportion of choices on the baited side of the arena that were to sucrosebaited pits was compared to chance; 50\%).

Subject rats also chose pits on the unbaited side of the arena less than expected by chance in all three trial types, regardless of whether they were tested together with good model rats, $t \mathrm{~s}(11) \geq 4.4$, or bad model rats, $t \mathrm{~s}(11) \geq 6.3$.

Additional analysis was restricted to the first choice that subject rats made during each trial. The first choice of each trial made by subject rats have special properties that render them of particular analytic interest. When making its first choice, the subject rat has no information from the outcome of earlier choices during the trial about the location of food (Bisbing et al., 2015). In addition, in the case of DR and DRMR trials, the model rat is present at the location of its second choice and has made exactly one earlier choice (to a pit where it is no longer present). Thus, the first choice made by the subject rat can be analyzed readily in terms of how the current or former choice of the model rat affects it.

Figure 6 shows the proportions of subject rats' initial choices in the same terms used to display all of their choices in the bottom panels of Fig. 5. There was no evidence that initial choices of the subject rats on the baited side of the arena 


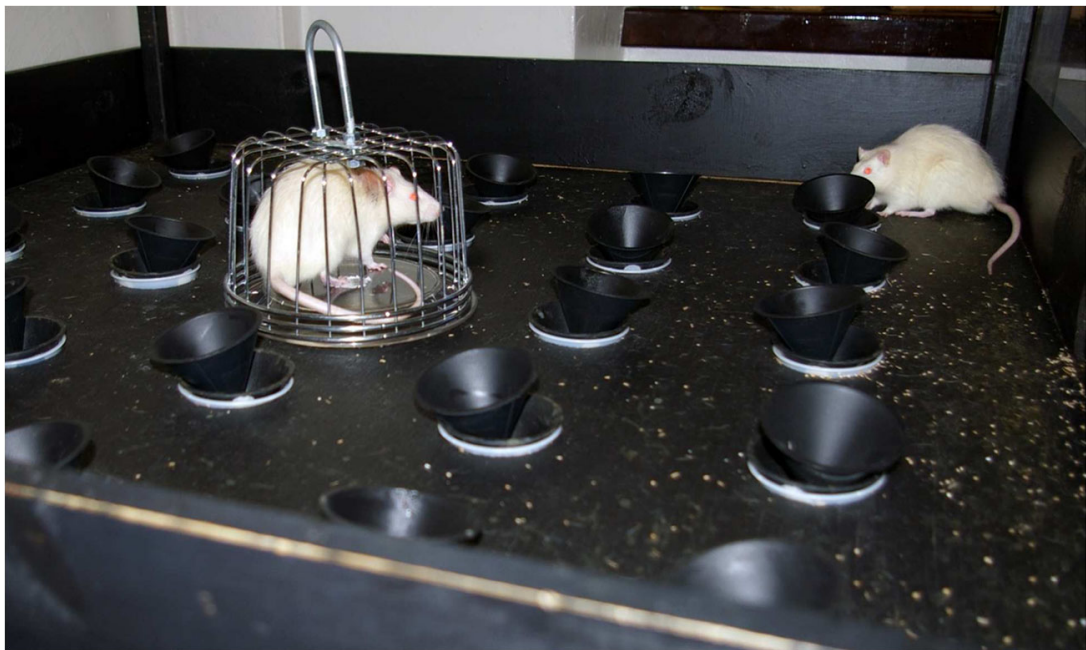

Fig. 4 Arena as it was configured in Experiment 2. The subject rat is restrained in the observer's cage as the model rat makes two choices

were more likely to pits baited with sucrose than expected by chance $(50 \%)$ in any of the three experimental conditions, regardless of whether they were tested with good or bad model rats, $t \mathrm{~s}(11)<2.0$.
These same choices by the subject rats were also analyzed in explicit terms of whether there was a tendency to visit pits that had been visited by the model rat. Figure 7 shows the proportion of the subject rats' first choices of each trial that

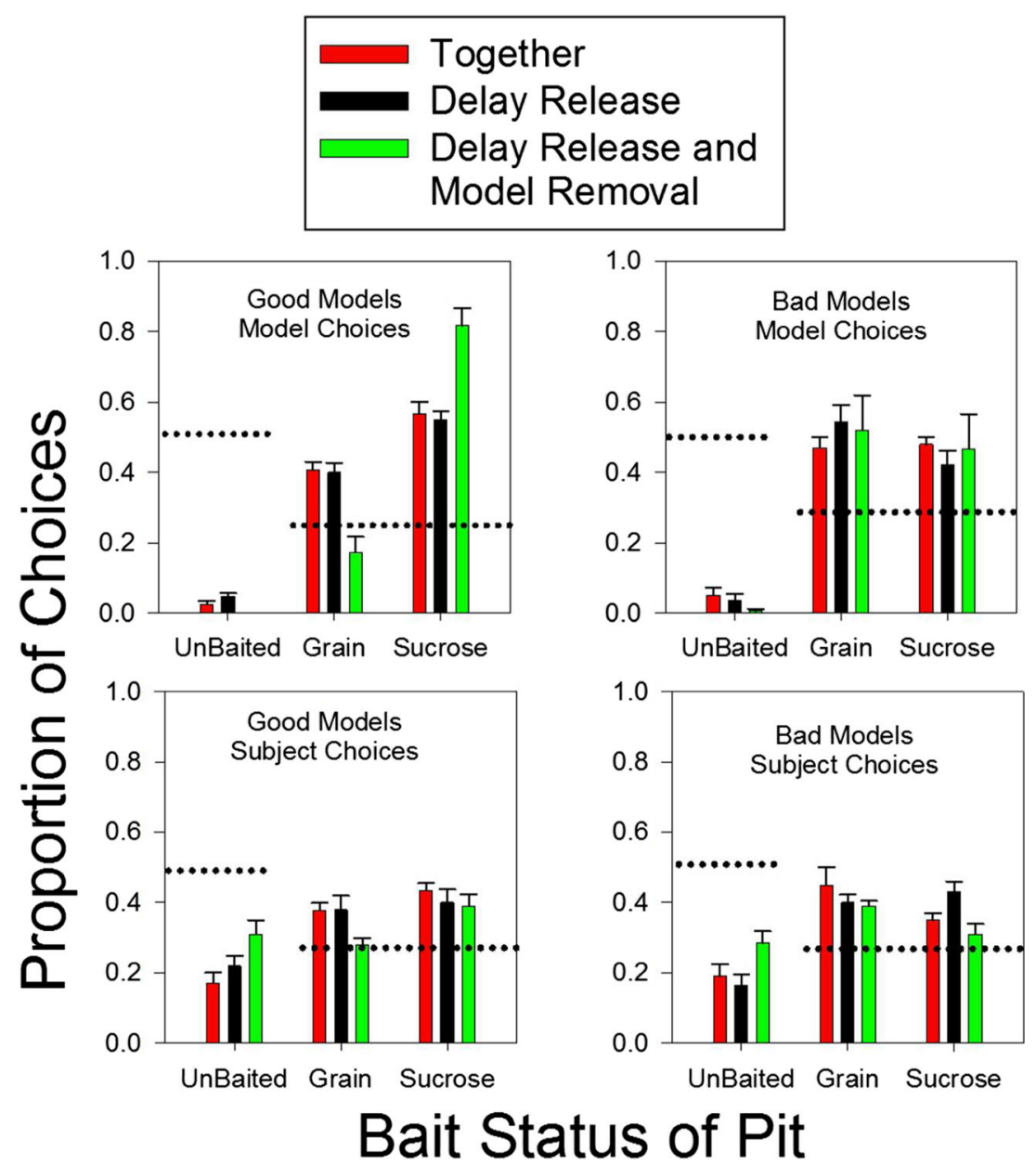

Fig. 5 Distributions of choices among pit types in the three conditions of Experiment 2 among good and bad model rats (top panels) and for subject rats when tested with good and bad model rats (bottom panels). Error bars show one standard error of the mean 


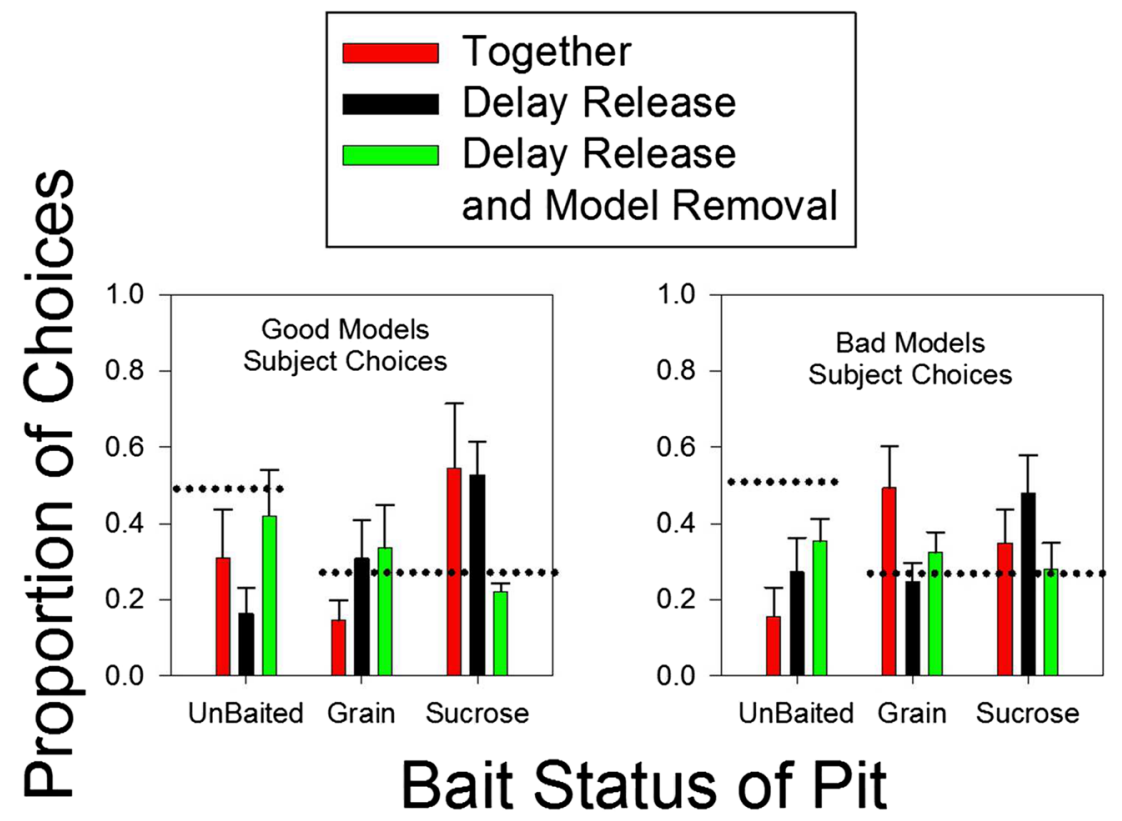

Fig. 6 Distributions of subject rats' initial choice of each trial among pit types in the three conditions of Experiment 2 when tested with good and bad model rats. Error bars show one standard error of the mean

were in each of three categories: choices of pits that had not been visited by the model rat earlier during the trial (labelled "UV" in the figure), choices of pits that were the most recent choice of the model rat (LV), and choices of pits that were chosen earlier in the trial by the model rat (PV). Remember that, in DR and DRMR trials, the subject rat was released from the observer cage as the model rat was visiting its second choice. Thus, there is exactly one pit chosen earlier in the trial by the model rat (the model rat's first choice) and one pit being chosen while the subject rat makes its first choice of the trial (the model rat's second choice). The proportions shown in Fig. 7 therefore can be evaluated in comparison to the proportions expected by chance (shown as reference lines in the figure). The obtained proportion of choices to the pit most recently chosen (LV) by either good or bad model rats exceeded chance in the together condition and in the DR condition, regardless of whether the model was good or bad, $t \mathrm{~s}(11)>4.4$. The obtained proportions of choices to the pit most recently chosen (LV) by either good or bad model rats did not differ from chance, $t \mathrm{~s}(11)<1.4$. Likewise, obtained proportions of choices by subject rats to the pit previously chosen by either a good or bad model rat (its first choice) did not differ from chance, $t \mathrm{~s}(11)<1.3$.

\section{Discussion}

The good model rat versus bad model rat distinction that was derived from performance in Experiment 1 continued to be valid in Experiment 2, in that good model rats, but not bad model rats, preferentially choose sucrose-baited locations over grain-baited locations. Unlike in Experiment 1, however, this was not reflected in different choice tendencies among the subject rats. There was no evidence that subject rats were more likely to choose sucrose-baited locations than grain-baited locations, regardless of whether they were tested following choices made by a good model rat or bad model rat.

There was, on the other hand, a substantial tendency for the first choice made by the subject rat after being released from the observer's cage to be to the location being chosen by the model rat. This tendency occurred in the together and DR trials but not in the DRR trials, indicating that the physical presence of the model rat is necessary to produce this effect. It should be pointed out, however, that disruption produced by removal of the model rat from the apparatus might also be a factor in the absence of the effect in the DRR trials. There was no tendency for the subject rats to visit locations that were chosen as the model rats' first choice. This is important because the model rats were no longer physically present at the location of its first choice when subject rats made the choices measured in this experiment, again suggesting the important of the model rat's physical presence for attracting choices by the subject rat.

In agreement with the results and conclusions from Experiment 1, the subject rats made choices to locations that had earlier been visited by the model rats. Also as in Experiment 1, they did so regardless of whether they were tested together with a model rat that preferentially chose sucrose locations over grain locations or a model rat that did not. However, the present experiment revealed that these social influences occurred only when the model rat was physically present at the chosen location. Thus, these results suggest that the proximal cue(s) controlling social influences on choices in 


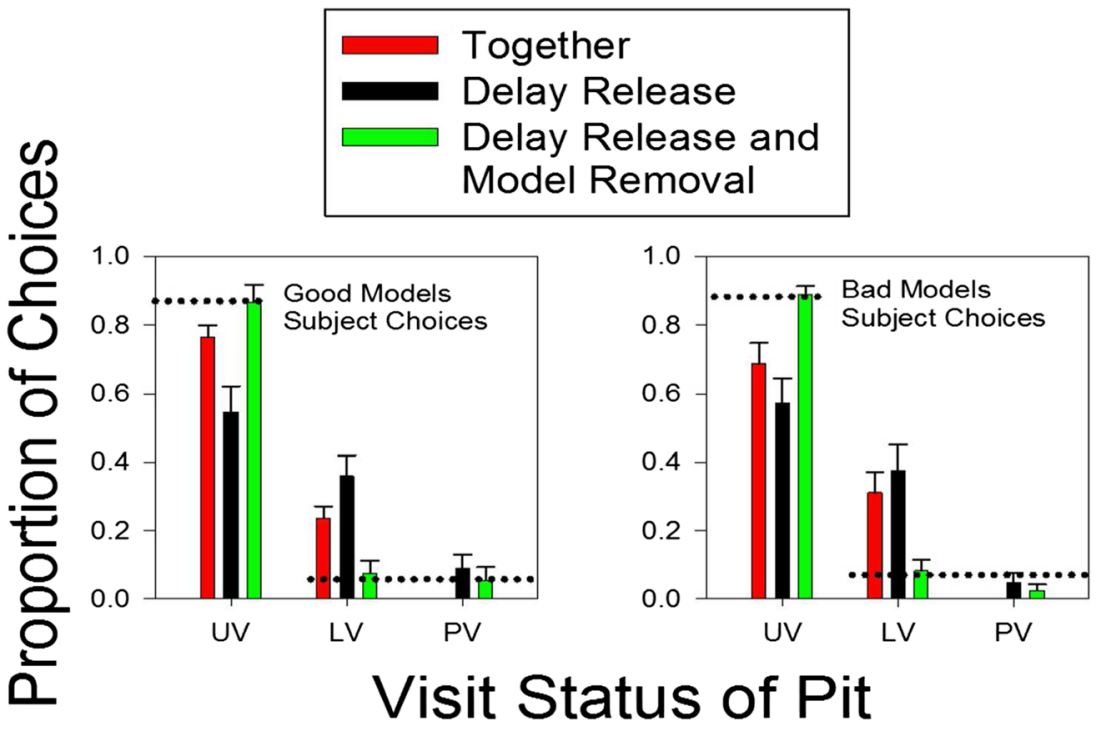

Fig. 7 Distributions of subject rats' initial choice of each trial among pits - as a function of whether the pit had been visited by the model rat - in the three conditions of Experiment 2 when tested with good and

this paradigm requires the physical presence of the model rat. This is consistent with data from the radial-arm maze, in which the physical presence of another rat produces affiliation even when previous visits to a maze location by another rat results in a tendency to avoid choice of that location (Brown, 2011).

\section{General discussion}

In these experiments, the choices made by model rats had an influence on the choices made by subject rats. In terms of choosing locations on the baited side of the arena rather than the unbaited side, subject rats acquired a tendency to search preferentially on the baited side based on the outcome of their own choices. The tendency was enhanced when a model rat was present. Thus, as in the earlier experiment of Brown et al. (2015), both personal information and social information controlled the arena side (or general area of the arena) in which subject rats made choices.

Also, as in the earlier experiment, only social information (in the form of choices made by the model rat) was available to distinguish among the locations on the baited side of the arena. In the earlier experiment, half of those locations were baited and half of them were not. The model rat choices resulted in subject rats preferentially choosing the specific locations that were baited. Similarly, in the present experiment, only social information had the potential to allow choice of sucrose-baited locations over grain-baited locations by the subject rats. It turned out, however, that that information was provided by only about half of the model rats, because the other half did not preferentially choose sucrose-baited locations over grain- bad model rats. UV = unvisited by model rat; $L V=$ last pit visited by model rat; $\mathrm{PV}=$ pits visited previously by the model rat. Error bars show one standard error of the mean

baited locations. Although we do not know what accounts for the difference across the two experiments, one reasonable possibility is simply that the difference in the value of locations baited with sucrose versus grain pellets is less than the earlier difference in the value of locations baited with sucrose versus nothing. This difference in the magnitude of the difference would be expected to produce a small social effect that was expressed to a measurable degree in some individual rats, but not in others.

Whatever the explanation, the fact that only some model rats demonstrated the location of sucrose locations (whereas other model rats did not) provided an opportunity to examine the specificity of the social influence demonstrated in this context. As outlined above, there is a continuum of possible specificity. At one extreme, a general social affiliation tendency would result in rats approaching conspecifics regardless of any information that their choices provide. In the radial-arm maze, rats tend to choose maze arms that are currently being chosen by another rat (or were just chosen by the other rat), and this occurs despite such maze arms being less likely to contain food than other maze arms (because the other rat just depleted the maze arm of food; Brown, 2011; Brown et al., 2007). This appears to be an example of a simple, nonspecific tendency to approach other rats.

On the other hand, social influence on spatial choices might result from the information provided by the other rat's choices. Cues corresponding to whether a rat finds food in a particular location when it makes a choice, or to the type/value of the food found there, would allow rats to modulate their choices in response to the food found in a particular location by a foraging partner. In addition, given that these rats are tested together with the same foraging partners repeatedly, it is possible that 
the subject rats might learn which model rats tend to choose sucrose-baited locations over grain-baited locations (or come to be controlled by cues corresponding to properties of the two kinds of model rats that they in common with each other).

The data support a mechanism of social influence that is in between these two extreme possibilities. Subject rats learn to make choices that model rats make apparently because, in their experience, the choices made by other rats are informative. However, they do not discriminate among model rats or among choices made by model rats, despite the fact that those cues are informative. Model rats, on the other hand, do not show any tendency to be influenced by subject rats. This is apparently because the choices of subject rats are not informative to model rats. Thus, a general social affiliation tendency is not sufficient to explain the social influence found in these experiments, because it cannot explain the asymmetry found between social influence on subject and model rats. Beyond determining whether a rat is or is not influenced by the choices of a conspecific, however, the social influences in these experiments are not very specific.

In previous experiments involving social influence in laboratory spatial choice tasks, including the one by Brown et al. (2015) that prompted the present experiments, it is not clear how specific the cues controlling social influences were, nor how flexibly rats could use those cues. The individual differences in model rats' tendencies to choose sucrose-baited locations in the present experiment provided an opportunity to address that issue and may provide an analytic approach that could be applied to other instances of social influence.

Author note Experiment 1 was the basis of a master's thesis submitted to Villanova University by Marie Saxon. The authors thank Julie Chen, Jessie Evans, and Christine Kurda for assistance conducting the experiments and Teagan Bisbing for technical advice.

\section{References}

Bisbing, T. A., Saxon, M., Sayde, J. M., \& Brown, M. F. (2015) Factors modulating social influence on spatial choice in rats, Journal of Experimental Psychology: Animal Behavior Processes, 41, 286300. doi:https://doi.org/10.1037/xan0000063
Brown, M. F. (2011). Social influences on rat spatial choice. Comparative Cognition \& Behavior Reviews, 6, 5-23. doi:https://doi.org/10. 3819/ccbr.2011.60003

Brown, M. F., Farley, R. F., \& Lorek, E. J. (2007). Remembrance of places you passed: Social spatial working memory in rats. Journal of Experimental Psychology: Animal Behavior Processes, 33, 213224. doi:https://doi.org/10.1037/0097-7403.33.3.213

Brown, M. F., Knight-Green, M. B., Lorek, E. J., Packard, C., Shallcross, W., Wifall, T., \& Schumann, E. (2008). Social working memory: Memory for the choices of another rat can increase or decrease spatial choice tendencies. Learning \& Behavior, 36, 327-340. doi: https://doi.org/10.3758/LB.36.4.327

Brown, M. F., Prince, T. M., \& Doyle, K. E. (2009). Social effects on spatial choice in the radial arm maze. Learning \& Behavior, 37, 269-280. doi:https://doi.org/10.3758/LB.37.3.269

Brown, M. F., Saxon, M. E., Bisbing, T., Evans, J., Ruff, J., \& Stokesbury, A. (2015). Five on one side: Personal and social information in spatial choice. Behavioural Processes, 112, 130-137. doi:https:// doi.org/10.1016/j.beproc.2014.12.012

Danchin, E., Giraldeau, L., Valone, T. J., \& Wagner, R. H. (2004). Public information: From nosy neighbors to cultural evolution. Science, 305, 487-491. doi:https://doi.org/10.1126/science.1098254

Galef, B. G., Dudley, K. E., \& Whiskin, E. E. (2008). Social learning of food preferences in 'dissatisfied' and 'uncertain' Norway rats. Animal Behaviour, 75, 631-637. doi:https://doi.org/10.1016/j. anbehav.2007.06.024

Galef, B. G., \& Whiskin, E. E. (2008). 'Conformity' in Norway rats? Animal Behaviour, 75, 2035-2039. doi:https://doi.org/10.1016/j. anbehav.2007.11.012

Giraldeau, L. A., \& Caraco, T. (2000). Social foraging theory. Princeton, NJ: Princeton University Press.

Giraldeau, L. A., Valone, T. J., \& Templeton, J. J. (2002). Potential disadvantages of using socially acquired information. Philosophical Transactions of the Royal Society of London B: Biological Sciences, 357, 1559-1566. doi:https://doi.org/10.1098/rstb.2002. 1065

Keller, M. R., \& Brown, M. F. (2011). Social effects on rat spatial choice in an open field task. Learning and Motivation, 42, 123-132. doi: https://doi.org/10.1016/j.lmot.2010.12.004

Laland, K.N. \& Plotkin, H.C.(1990) Social learning and social transmission of foraging information in Norway rats (Rattus norvegicus). Animal Learning \& Behavior, 18, 246-251. https://doi.org/10. 3758/BF03205282

Rieucau, G., \& Giraldeau, L. A. (2011). Exploring the costs and benefits of social information use: An appraisal of current experimental evidence. Philosophical Transactions of the Royal Society of London B: Biological Sciences, 366, 949-957. doi:https://doi.org/10.1098/ rstb.2010.0325

Vickery, W. L., Giraldeau, L., Templeton, J. J., Kramer, D. L., \& Chapman, C. A. (1991). Producers, scroungers, and group foraging. The American Naturalist, 137, 847-863. doi:https://doi.org/10. $1086 / 285197$

Ward, A., \& Webster, M. (2016). Sociality: The behaviour of group-living animals. Basel, Switzerland: Springer. 